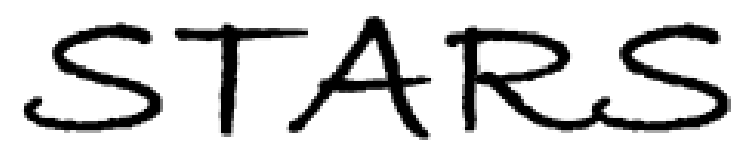

University of Central Florida

STARS

$1-1-2004$

\title{
Arbitrary angle waveguiding applications of two-dimensional curvilinear-lattice photonic crystals
}

Javad Zarbakhsh

Frank Hagmann

Sergei F. Mingaleev

Kurt Busch

University of Central Florida

Kurt Hingerl

Find similar works at: https://stars.library.ucf.edu/facultybib2000

University of Central Florida Libraries http://library.ucf.edu

This Article is brought to you for free and open access by the Faculty Bibliography at STARS. It has been accepted for inclusion in Faculty Bibliography 2000s by an authorized administrator of STARS. For more information, please contactSTARS@ucf.edu.

\section{Recommended Citation}

Zarbakhsh, Javad; Hagmann, Frank; Mingaleev, Sergei F.; Busch, Kurt; and Hingerl, Kurt, "Arbitrary angle waveguiding applications of two-dimensional curvilinear-lattice photonic crystals" (2004). Faculty Bibliography 2000s. 4916.

https://stars.library.ucf.edu/facultybib2000/4916

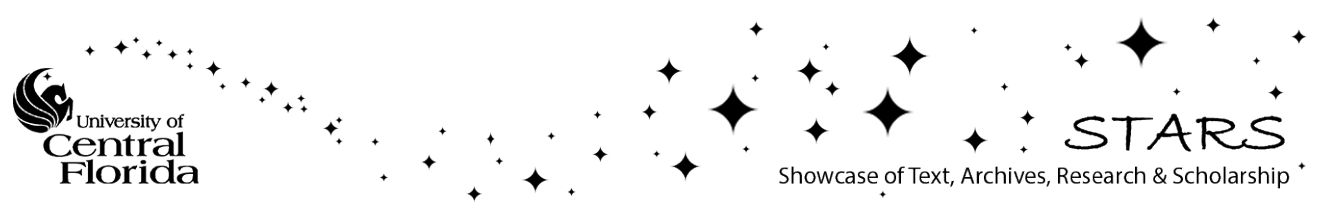




\title{
Arbitrary angle waveguiding applications of two-dimensional curvilinear-lattice photonic crystals
}

\author{
Javad Zarbakhsh \\ Christian Doppler Labor für Oberfächenoptische Methoden, Institut für Halbleiter und Festkörperphysik, \\ Universität Linz, A-4040 Linz, Austria \\ Frank Hagmann \\ Institut für Theorie der Kondensierten Materie, Universität Karlsruhe, 76128 Karlsruhe, Germany \\ Sergei F. Mingaleev \\ Institut für Theorie der Kondensierten Materie, Universität Karlsruhe, 76128 Karlsruhe, Germany \\ and Bogolyubov Institute for Theoretical Physics, 03143 Kiev, Russia \\ Kurt Busch \\ Institut für Theorie der Kondensierten Materie, Universität Karlsruhe, 76128 Karlsruhe, Germany \\ and Department of Physics and School of Optics, CREOL and FPCE, University of Central Florida, \\ Orlando, Florida 32816 \\ Kurt Hinger ${ }^{\text {a) }}$ \\ Christian Doppler Labor für Oberflächenoptische Methoden, Institut für Halbleiter and Festkörperphysik, \\ Universität Linz, A-4040 Linz, Austria
}

(Received 23 February 2004; accepted 22 April 2004; published online 19 May 2004)

\begin{abstract}
We introduce a fresh class of photonic band-gap materials, curvilinear-lattice photonic crystals, whose distinctive feature is that their individual scatterers are arranged in a curvilinear lattice. We show that adhering to some restrictions in the acceptable lattice transformations, one can achieve omnidirectional photonic band gaps for an entire subclass of such structures. We demonstrate, designing an efficient arbitrary-angle waveguide bend, that curvilinear-lattice photonic crystals can be employed for creation of original types of nanophotonic devices. (C) 2004 American Institute of Physics. [DOI: 10.1063/1.1760222]
\end{abstract}

One of the most interesting and important achievements of modern optics is the discovery that the density of photonic states in some artificially manufactured optical materials can be controlled in a dramatic fashion-right up to the complete vanishing of photonic states in certain intervals of wavelengths, the so-called photonic band gaps. ${ }^{1}$ Such materials are widely known as photonic band gap (PBG) materials or photonic crystals (PCs). The last term reflects the fact that commonly studied designs of PBG materials are based on periodic modulation of the refractive index in two or three spatial directions. Investigations of perfectly periodic structures are historically favored by the analogy to the opening of electronic band gaps in atomic and molecular crystals. Due to the periodicity of these structures, the spectrum of electronic states is organized into an infinite series of electronic bands, with a possibility to open gaps between them. The same holds for the spectra of photonic modes inside materials with sufficiently high-contrast periodic modulation of the refractive index.

For a long time, structural periodicity has been considered as a vital force for opening various types of band gaps. However, after the experimental discovery of electronic quasicrystals in $1984,{ }^{2}$ it has been ascertained that the dominant role in opening band gaps is played by the short-range periodicity. Recent investigations of photonic quasicrystals

${ }^{a)}$ Electronic mail: kurt.hinger1@jku.at
$(\mathrm{PQCs})^{3-8}$ have substantiated the validity of this assertion in the case of PBG materials.

Based on this analogy with atomic and molecular crystals and quasicrystals, researchers have to date limited their study to only three basic ordering types of photonic structures: crystalline, quasicrystalline, and amorphous (assuming in the latter case the presence of some level of disorder). However, in the case of artificially manufactured PBG materials there is no inherent limitation for these restrictions. As a result, other sophisticated forms of ordering may also lead to PBGs and the resulting structures may exhibit advantages over existing PC and PQC structures. A profound theoretical discussion, employing the scaling theories of light localization to discuss the corresponding different coherence length scales has been given by John. ${ }^{9}$

In this letter we introduce a fresh subclass of PBG materials, which we name curvilinear-lattice photonic crystals. Their distinctive feature is that individual scatterers are arranged in a curvilinear lattice. As an illustration, we explicitly discuss the example of a two-dimensional photonic crystal formed by a system of silicon cylinders (with refractive index $n=3.4$ ) situated in an air background. For the sake of simplicity, we assume hereafter that all cylinders have the same radius $r=0.16 a$, where $a$ is an average distance between nearest-neighbor cylinders. The standard arrangements of cylinders that are studied with respect to the opening of photonic band gaps, include square lattices [shown in Fig. 1(a)], triangular lattices [shown in Fig. 1(b)], and various types of quasicrystalline lattices. ${ }^{3-8}$ In all cases, there exists 


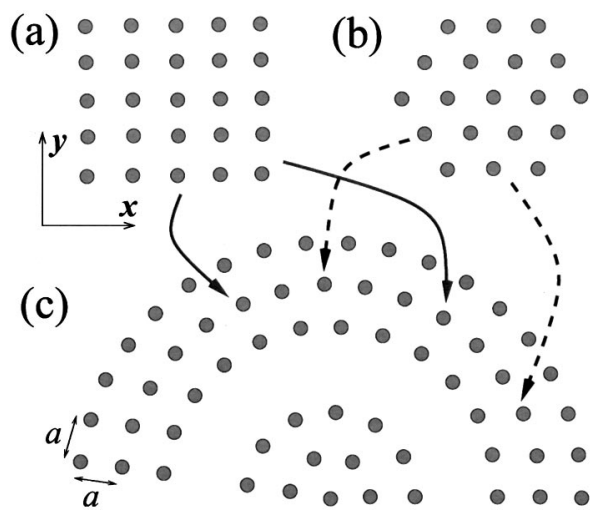

FIG. 1. Top views of photonic structures with different arrangements of dielectric cylinders: (a) square-lattice structure; (b) triangular-lattice structure; (c) curvilinear-lattice structure with an embedded waveguide which allows one to construct an arbitrary-angle waveguide bend. Due to the construction principle, the local symmetry of the curvilinear lattice transforms between an almost-square (indicated by full arrows) and an almosttriangular (indicated by dashed arrows) lattices.

a large complete photonic band gap for $E$-polarized light (i.e., with the electric field parallel to cylinders). It is very intriguing to note that the spectral position of the PBG for our choice of cylinders is almost unchanged for different types of lattices: It lies in the wavelength interval $1.95 a<\lambda$ $<3.15 a$ for the triangular lattice and in the interval $2.15 a$ $<\lambda<3.1 a$ for the square lattice.

This fact brings up the question: Which other stretching and shearing lattice transformations keep the band gap in the same frequency interval? To clarify this issue, we present in Fig. 2 the band-gap map for various uniform lattice transformations in the systems of periodically arranged dielectric rods. We consider shearing transformation which vary the angle $\phi$ between the lattice vectors $\mathbf{a}_{1}$ and $\mathbf{a}_{2}$ within the interval $60^{\circ}<\phi<90^{\circ}$, and the stretching transformations leading to variations in the lattice constant $a$ that do not exceed about $15 \%$. This map demonstrates that the fundamental band gap for $E$-polarized light is retained in the interval of wavelengths $2.15 a \leqq \lambda \lesssim 3.1 a$ for all lattice transformations considered. Therefore, one can expect that any smooth transformations which keep the distance between nearest-neighbor cylinders close to $a$ should retain the pho-

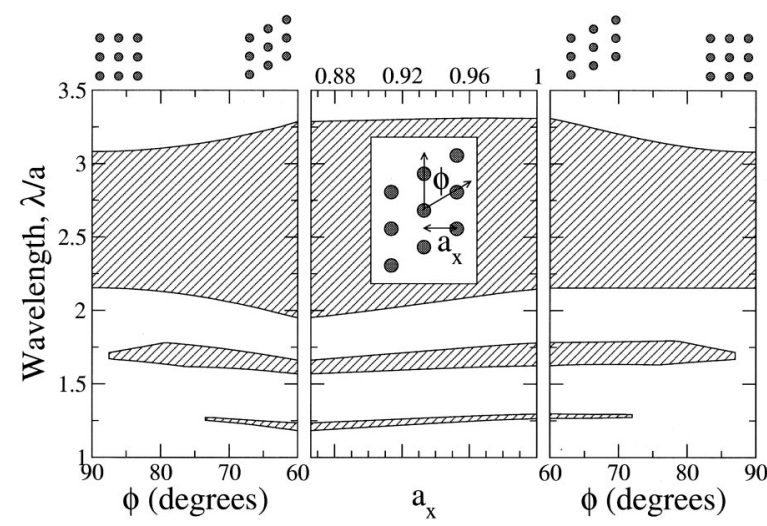

FIG. 2. Photonic band-gap map for $E$-polarized light in a perfectly periodic PC made of dielectric rods (with radius $r=0.16 a$ and refractive index $n=3.4)$ under various uniform lattice transformations, calculated by BANDSOLVE (Ref. 12). The fundamental band gap is retained in the wavelength interval $2.15 a \lesssim \lambda \lesssim 3.1 a$ for all lattice transformations presented here.

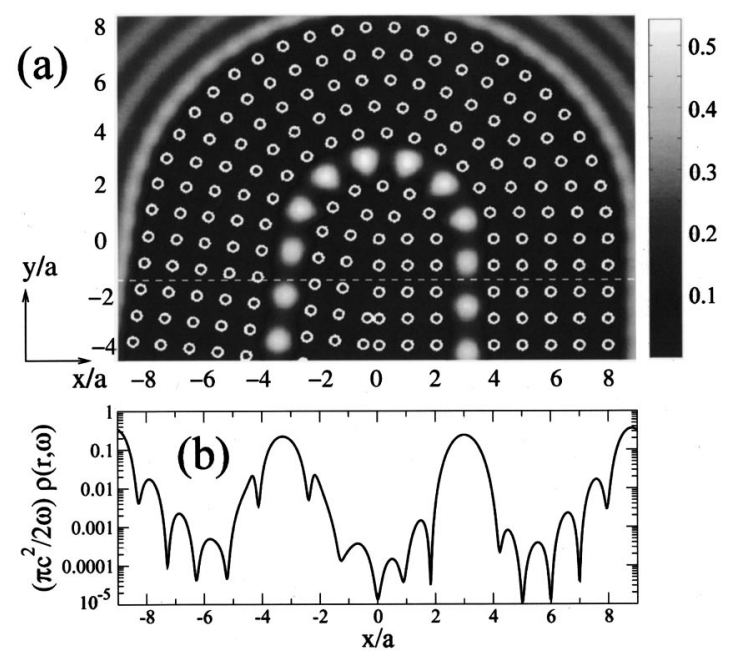

FIG. 3. (a) Local density of states (LDOS) calculated for $\lambda=2.5 a$ for the curvilinear-lattice structure with embedded waveguide shown in Fig. 1(c); (b) LDOS profile along the line marked in (a) by the dashed white line.

tonic band gap in the entire curvilinear-lattice PC.

One example of such an "allowed" curvilinear-lattice PC structure is shown in Fig. 1(c). Here, the cylinders are positioned at a sequence of circles with radii varying from $a$ to $6 a$, keeping the radial and tangential distances between cylinders close to $a$. The rods in the third circle are carved out, creating a waveguide with an arbitrary-angle waveguide bend. Due to the construction algorithm, each local surrounding in Fig. 1(c) is very close to one of the lattices considered in Fig. 2. A careful inspection of Fig. 1(c) reveils several smooth transitions between regions of an almostsquare lattice (e.g., those indicated by full arrows) and regions of an almost-triangular lattice (e.g., those indicated by dashed arrows).

However, one can harbor natural doubts as to whether the "smooth" transitions between square and hexagonal lattices in Fig. 1(c) are really smooth for accepting the argumentation based on the analysis of Fig. 2. A crude estimate for this moot point is provided by the localization criterion: ${ }^{1,9}$ The penetration depth $\lambda_{\text {env }}$ of the electromagnetic field into the photonic crystal can be calculated within a parabolic approximation of the bands near the PBG as $\lambda_{\text {env }}=\left|\alpha /\left(\omega_{\text {edge }}-\omega\right)\right|^{1 / 2}$. Here, $\omega=(2 \pi c / \lambda)$ is the frequency of light inside a photonic band gap whose band edge is at $\omega_{\text {edge }}$, and $\alpha=\left.\left(\partial^{2} \omega / \partial k^{2}\right)\right|_{\mathbf{k}_{\text {edge }}}$ is the inverse effective photonic mass at the band edge with wavevector $\mathbf{k}_{\text {edge }}$ $=\mathbf{k}\left(\omega_{\text {edge }}\right)$. Finally, $c$ is the speed of light in vacuum. The calculations for the square and triangular lattices predict that the field penetration depth at the center of the band gap is less than $0.75 a$. Therefore, although the lattice transitions exploited in Fig. 1(c) occupy only about two lattice constants, they may be considered as sufficiently smooth.

Of course, such semiquantitative arguments need to be substantiated by a quantitative assessment for the quality of PBGs inside nonperiodic structures. This assessment can be most naturally provided by calculating the local density of states (LDOS), $\rho(\mathbf{r}, \omega)$, which has been recently employed, in particular, for studies of complete PBGs in PQCs. ${ }^{8}$ In Fig. 3 we present LDOS calculations for our curvilinear-lattice waveguiding structure that are based on the numerically ex- 


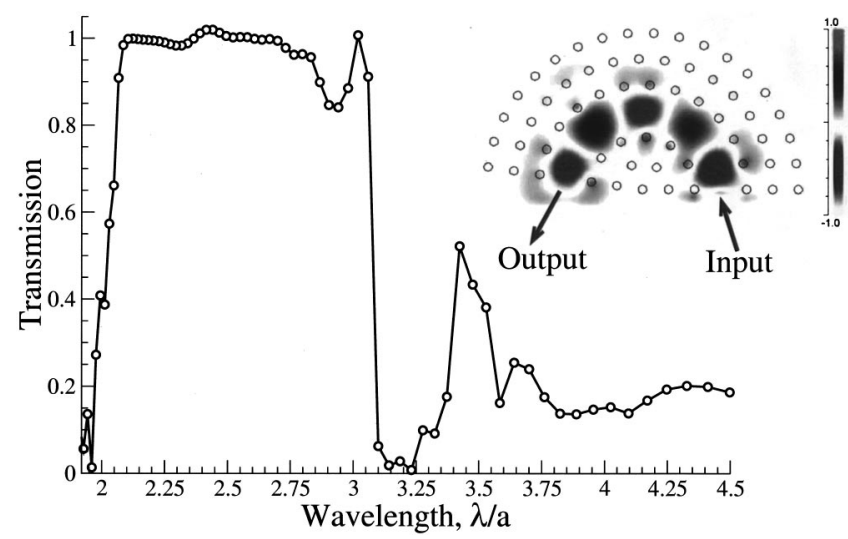

FIG. 4. Transmission spectrum for the waveguide bend embedded into the curvilinear-lattice photonic crystal as it is shown in Fig. 1(c). In the inset we plot the distribution of the electric field at $\lambda=2.5 a$.

act multiple multipole expansion technique. ${ }^{10}$ The LDOS is indeed very small everywhere inside the curvilinear-lattice $\mathrm{PC}$ except for its waveguiding region.

One of the most attractive applications of PCs is their usage for the creation of high-density integrated photonic circuits. Consequently, it is very important to verify that the curvilinear-lattice structures suggested in this letter can be used for an efficient guidance of light. In Fig. 4 we plot the results of finite difference time domain $(\text { FDTD })^{11}$ calculations for the transmission spectrum of $E$-polarized light through the curvilinear waveguiding structure shown in Fig. 1(c). These simulations have been performed using the FULLWAVE commercial software ${ }^{12}$ with spatial and temporal discretization steps of $0.01 a$ and $0.007 a / c$, respectively. In the inset to Fig. 4, we plot the electric field distribution at wavelength $\lambda=2.5 a$, for which the power transmission is almost perfect. As we see, in spite of the absence of a long-range periodicity in this structure, it guides an $E$-polarized light very efficiently: the transmission coefficient is close to unity over a very broad interval of wavelengths, $2.1 a \leqslant \lambda \leqslant 3 a$.

In conclusion, we have introduced a fresh subclass of PBG materials-curvilinear-lattice photonic crystals. We have shown that when adhering to some restrictions in the lattice transformations, one can achieve omnidirectional PBGs within these structures. The corresponding restrictions can be derived from the analysis of the band-gap maps for stretching and shearing transformations (similar to what we presented in Fig. 2) together with an analysis of the corresponding localization lengths. Obviously, these restrictions are different for different PBGs and different geometries of the individual scatterers, and extensive further investigations are still required to determine the most successful parameters for practical applications.

From our point of view, the most interesting applications of the curvilinear-lattice PCs are related to the creation of ultracompact integrated PC circuits. Until now such circuits are usually designed by embedding into PCs various types of defects, while leaving the local lattice symmetry untouched. This approach has been successfully applied to designing of many simple PC devices such as waveguide bends, splitters, and so forth. However, to be successful this approach often involves embedding additional defects with very small radii ${ }^{13}$ which are difficult to fabricate with acceptable accuracy. In contrast, our approach of using curvilinear transformations of a PC lattice provides designers with an additional degree of freedom which can be exploited in numerous ways, e.g., for avoiding the usage of small-size defects. Moreover, as we demonstrate in Fig. 4 by the example of an efficient arbitrary-angle waveguide bend, the curvilinearlattice PCs can also be employed for creation of original types of nanophotonic devices. In addition to arbitrary-angle waveguide bends and splitters, they can include various types of topological defects, arbitrary-radius circular resonators, whispering gallery mode devices, etc.

The authors are grateful to Johann Messner from the Linz Supercomputer Department for numerous support as well as CPU time grants and to Heinz Seyringer from Photeon Technologies. F.H., S.F.M., and K.B. acknowledge support by the Center for Functional Nanostructures (CFN) of the Deutsche Forschungsgemeinschaft (DFG) within the Project A 1.2. The research of K.B. is further supported by the DFG under Grant No. Bu 1107/2-3 (Emmy-Noether Program).

${ }^{1}$ J. D. Joannopoulos, R. D. Meade, and J. N. Winn, Photonic Crystals: Molding the Flow of Light (Princeton University Press, Princeton, NJ, 1995).

${ }^{2}$ D. Shechtman, I. Blech, D. Gratias, and J. W. Cahn, Phys. Rev. Lett. 53, 1951 (1984).

${ }^{3}$ Y. S. Chan, C. T. Chan, and Z. Y. Liu, Phys. Rev. Lett. 80, 956 (1998).

${ }^{4}$ T. Janssen, Phys. Rep. 168, 55 (1988).

${ }^{5}$ C. Jin, B. Cheng, B. Man, Z. Li, D. Zhanga, S. Ban, and B. Sun, Appl. Phys. Lett. 75, 1848 (1999).

${ }^{6}$ M. E. Zoorob, M. D. B. Charlton, G. J. Parker, J. J. Baumberg, and M. C. Netti, Nature (London) 404, 740 (2000).

${ }^{7}$ M. A. Kaliteevski, S. Brand, R. A. Abram, T. F. Krauss, P. Millar, and R. M. De la Rue, J. Phys.: Condens. Matter 13, 10459 (2001).

${ }^{8}$ Y. Wang, B. Cheng, and D. Zhang, J. Phys.: Condens. Matter 15, 7675 (2003).

${ }^{9}$ S. John, Confined Electrons and Photons, edited by E. Burstein and C. Weisbuch (Plenum, New York, 1995), p. 523; Photonic Band gaps and Localization, edited by C. M. Soukoulis (Plenum, New York, 1993), p. 1.

${ }^{10}$ A. A. Asatryan, K. Busch, R. C. McPhedran, L. C. Botten, C. M. de Sterke, and N. A. Nicorovici, Waves Random Media 13, 9 (2003).

${ }^{11}$ A. Taflove and S. C. Hagness, Computational Electrodynamics: The Finite-Difference Time-Domain Method (Artech House, Boston, 2000).

${ }^{12} \mathrm{http} / / /$ www.rsoftdesign.com

${ }^{13}$ A. Chutinan, M. Okano, and S. Noda, Appl. Phys. Lett. 80, 1698 (2002), and references therein. 